\title{
Co-designing Socially Assistive Sidekicks for Motion-based AAC
}

\author{
Stephanie Valencia ${ }^{1}$, Michal Luria $^{1}$, Amy Pavel $^{1}$, Jeffrey P. Bigham ${ }^{1}$, Henny Admoni $^{2}$ \\ ${ }^{1} \mathrm{HCI}$ Institute and ${ }^{2}$ Robotics Institute, Carnegie Mellon University \\ \{svalenci,mluria,apavel,jbigham\}@cs.cmu.edu,henny@cmu.edu
}

\begin{abstract}
Augmentative and alternative communication (AAC) devices enable speech-based communication. However, AAC devices do not support nonverbal communication, which allows people to take turns, regulate conversation dynamics, and express intentions. Nonverbal communication requires motion, which is often challenging for AAC users to produce due to motor constraints. In this work, we explore how socially assistive robots, framed as "sidekicks," might provide augmented communicators (ACs) with a nonverbal channel of communication to support their conversational goals. We developed and conducted an accessible co-design workshop that involved two ACs, their caregivers, and three motion experts. We identified goals for conversational support, co-designed prototypes depicting possible sidekick forms, and enacted different sidekick motions and behaviors to achieve speakers' goals. We contribute guidelines for designing sidekicks that support ACs according to three key parameters: attention, precision, and timing. We show how these parameters manifest in appearance and behavior and how they can guide future designs for augmented nonverbal communication.
\end{abstract}

\section{KEYWORDS}

communication, nonverbal behavior, socially assistive robots

\section{ACM Reference Format:}

Stephanie Valencia ${ }^{1}$, Michal Luria ${ }^{1}$, Amy Pavel ${ }^{1}$, Jeffrey P. Bigham ${ }^{1}$, Henny Admoni $^{2}$. 2021. Co-designing Socially Assistive Sidekicks for Motion-based AAC. In Proceedings of the 2021 ACM/IEEE International Conference on Human-Robot Interaction (HRI '21), March 8-11, 2021, Boulder, CO, USA. ACM, New York, NY, USA, 10 pages. https://doi.org/10.1145/3434073.3444646

\section{INTRODUCTION}

Speech generating devices, a type of augmentative and alternative communication (AAC) system that is often used by individuals with speech and motor disabilities, produce synthetic speech from a user's selection of letters, words, or images on a tablet or computer [7]. Speaking using an AAC device usually takes longer due to message composition delays, with rates ranging from 3-10 words per minute [23, 27]. Even though AAC systems enable communication, verbal speaking partners (non-AAC speakers) often negatively impact the interaction. For example, when they communicate with an augmented communicator (AC) who uses an AAC device they often do not allow enough time for communication, or lack familiarity with an AC's communication style [21, 45, 46]. Partners may

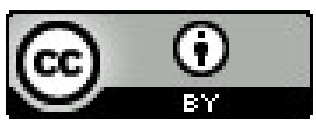

This work is licensed under a Creative Commons Attribution International 4.0 License.

HRI '21, March 8-11, 2021, Boulder, CO, USA.

(C) 2021 Copyright held by the owner/author(s).

ACM ISBN 978-1-4503-8289-2/21/03.

https://doi.org/10.1145/3434073.3444646

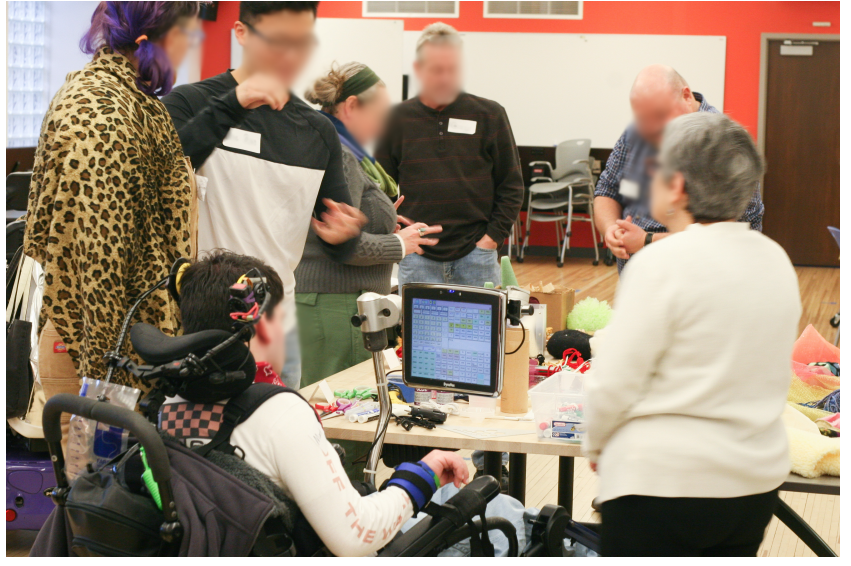

Figure 1: We explore the metaphor of an "assistive sidekick" to design robots that assist augmented communicators by playing a supporting role through nonverbal behavior.

also limit ACs' opportunities to participate in conversation, for instance by asking only yes/no questions, when ACs want to reply to open-ended ones; by dominating the conversation; or by failing to respond to ACs' communication attempts [16, 22, 58].

Speaking speed is not the only reason for difficulties in bridging communication between augmented and non-augmented speakers. Augmented communicators are commonly constrained in their ability to use nonverbal behaviors. Nonverbal behaviors have been shown to support conversations, for example by providing partners with feedback, by securing speaking turns, or by accompanying spoken language to aid in interpretation [20,28, 37]. Verbal speakers use many nonverbal behaviors that can be difficult for ACs with motor disabilities to express. Augmented communicators often do use a range of nonverbal behaviors (e.g., facial gestures, body orientation) but these are hard to interpret by others [12, 46, 58].

In Human-Robot Interaction (HRI), social robots have used motion to support people in a variety of related communication contexts through nonverbal behavior. For example, researchers have used social robot gaze to facilitate turn-taking [2, 41], and robot body gestures to mediate interpersonal conflict [24, 57]. In this work, we leverage robots' advantages of embodiment and motion for nonverbal communication in the domain of AAC, to explore how social robots could be designed to support ACs' nonverbal communication. We use the metaphor of "assistive sidekick" to explore robot designs that support ACs, rather than function as independent agents.

To investigate how social robots could be used in augmented conversations, we conducted an accessible, multi-phase co-design workshop, with the goal of designing nonverbal behaviors that are rich and understandable enough to support augmented speakers' 
conversational goals. Participants in the workshop included ACs and their caregivers, also referred to as close conversation partners, in order to set the co-design process around their experiences. It also included puppeteers, who contributed their expertise in expressive motion to explore new motion-based behaviors for assistive robots.

Our work makes three primary contributions to socially interactive robots for accessibility and HRI design research. First, we offer an example of a co-design workshop adapted to include the participation of ACs with motor disabilities, who are often excluded from design processes. Second, based on the workshop process and outcomes, we contribute guidelines for designing social robots that support ACs through expressive nonverbal behavior. We describe these guidelines according to three key aspects: attention, precision, and timing in conversation. Third, we identify a list of potential conversational goals important to ACs that "assistive sidekicks" could support, and a set of motions and behaviors to achieve them.

\section{BACKGROUND AND RELATED WORK}

Our work builds on prior research on (1) augmenting AAC conversations, (2) nonverbal communication, (3) peripherally interactive robots, and (4) accessibility in participatory design.

\subsection{Augmenting AAC Conversations}

Speech-generating AAC devices provide a way to communicate and convey information, but there is still a high rate of reported breakdowns and miscommunication in conversations between people with a speech disability and those without [21, 56, 58]. To improve conversation dynamics for augmented communicators, prior work has considered adding communication modalities to AAC systems. Personal voices have been used to convey affect and emotion [15, 44]. Other research efforts have used a mobile companion app [16], partner-facing awareness displays, and physical LED clusters [54] to increase a partner's awareness by making an AC's activity visible (e.g., listening, typing, or resting). Using nonverbal symbols to support communication (e.g., LED clusters) revealed that more abstract symbols, such as color change, may be difficult to interpret. In contrast, other explored modes (e.g., voice, screen) may fail to draw the needed attention. To address such challenges, we consider motion as an alternative and underexplored communication modality to support ACs. In addition, our work prioritizes increasing awareness among unfamiliar partners who do not know the AC or are new to AAC-based conversation by leveraging socially-recognizable nonverbal behaviors.

\subsection{Nonverbal and Embodied Communication}

Embodied interaction refers to the way our perception of physical and social phenomena develops in interplay with our space and surroundings [14, 19]. It leverages people's ability to interpret nonverbal behavior to communicate a range of ideas and emotions through motion [1, 52]. Gaze, body orientation, and pointing gestures are all examples of nonverbal communication that can support directing attention to different speakers in a group conversation [28, 53] and are especially powerful in face-to-face interactions. A shared physical environment allows the use of specific spatial cues such as referencing an object or orienting the body or gaze towards a space of interest to establish common ground and joint attention [4].
One of the prominent qualities that makes robots unique is their embodiment in physical space. Robots can not only manipulate objects in space, but also express complex interactions through motion [1, 11, 29]. In our work we focus on exploring how robots' nonverbal communication and embodied interaction can support $\mathrm{AC}$ participation while maintaining their agency in conversation.

\subsection{Peripheral Social Robots in Conversation}

Prior work has explored the use of peripheral interactive objects as communication supporters in a range of domains. One of the defining features of peripheral interfaces is that they exist primarily in the background and are only called to the foreground when necessary-they are not themselves interaction partners [25, 35]. In HRI, the notion of peripheral robots has also been explored. For example, a peripheral robotic device that draws attention when the conversation between a romantic couple becomes aggressive [24], one that shapes conversational dynamics in groups [57] or accompanies text messaging [43]. Peripheral and embodied devices have also been suggested as socially assistive robots. Minimally expressive anthropomorphic robots have been used to support autistic children's communication [47] and to facilitate interaction between people with dementia and caregivers [40].

In addition, social robots have the quality of being able to take on a specific social role in a conversation, such as an over-hearer [30] or a facilitator [36]. Prior work has suggested a metaphor of sidekicks as a way to think about social robots [34] that support a "protagonist" by existing as a "secondary entity" and appearing only when needed. We build on this framing to design peripheral robot sidekicks that increase augmented communicators' agency in conversation through nonverbal behavior. We explore robot designs that support the AC, rather than function as independent agents.

\subsection{Accessible Participatory Design}

Our workshop design draws from accessible participatory design (PD). PD highlights the participation of diverse stakeholders, including non-designers who may directly influence the design process and/or outcomes [13, 48, 49]. Previous work on accessible PD has included co-designing robots with older adults with depression [32], interactive toys with children with autism spectrum disor$\operatorname{der}[17,55]$, robot-based navigation with people with visual impairments [3], and tool exploration with people with aphasia [18, 31, 39]. However there are few examples of including augmented communicators who have motor disabilities in ideation and co-creation of robots and other technologies [5]. In our work we developed a co-design workshop [9] to place augmented communicators in the center of the process of envisioning robotic sidekicks as a future communication technology they might use.

\section{METHOD}

We conducted our research in two steps (Figure 2) to answer the following research questions: (1) What are ACs' nonverbal needs in conversation?; (2) How, if at all, can a sidekick support ACs in conversation? The first step consisted of gathering information about current ACs' needs in conversation via an online survey distributed to a group of ACs. The answers collected in the online survey served to identify interaction challenges a sidekick could 


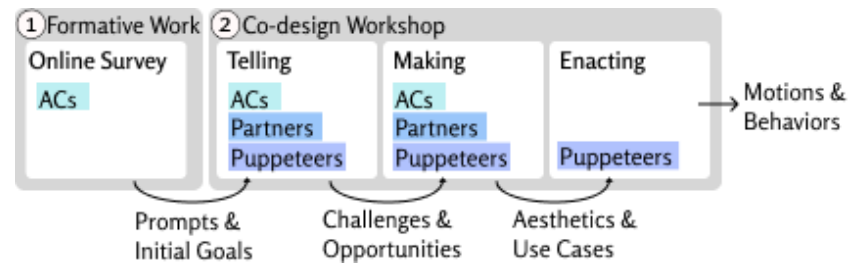

Figure 2: Our two step process included formative work and a three-phase co-design workshop. Participants included augmented communicators (ACs), their close conversational partners, and professional puppeteers.

help with. The second step of our process consisted of an in-person co-design workshop split into three phases: Telling, Making, and Enacting [9]. Telling activities focused on scaffolding the sharing of stories of current lived experiences, reflecting on what mattered to ACs in communication, and identifying potential challenges to address. Making consisted of externalizing ideas and embodying solutions in the form of physical artifacts to define possible sidekick forms and aesthetics. Lastly, puppeteers enacted and explored sidekick motions through behavior improvisations.

\subsection{Step 1: Online Survey}

Four augmented communicators (aged 33 to 54) with motor disabilities responded to our survey. Respondents use switches, eye gaze, touch screens, and keyboards to access their AAC devices, and all have more than 5 years of experience using AAC. The survey included a combination of Likert scale and open-ended questions asking participants to rate how often they experienced specific situations in conversation and to describe them further.

Three of four participants indicated that they participate in conversations less than they desire to. Participants expressed not being able to communicate fast enough to keep up with topic transitions, a finding that confirms prior work [45, 58]. Participants reported feeling pressure to respond quickly in an ongoing conversation and often asking their caregivers to explain things on their behalf.

Participants also shared that people who are close to them occasionally help facilitate their communication by telling others to be patient, to let them participate, and to address them directly ("They tell [people] to talk to me", "[I like that] my partner looks [at] other people to make sure they are communicating with me"), "It's nice to have someone who knows I am typing and will tell everyone." Sometimes close conversation partners use hand gestures to make another person wait until the AC finishes their thought ("They look at me the whole time with their pointer finger extended out, as if she is saying [to the other person], 'Hold On'.)"

Based on the online survey and along with prior work [58], we identified that it would be worthwhile to explore whether an embodied technology could play a supportive role similar to a knowledgeable conversation partner and communicate that (1) an AC would like to participate, (2) that they should be addressed directly, or (3) that they are composing a message.

We consulted with a speech-language pathologist (SLP) who confirmed the identified challenges as known problems, and suggested other critical tasks partners help with, such as telling others that an $\mathrm{AC}$ is having trouble with their device. We used these findings to create an initial list of goals a sidekick could support (Table 1).

We framed the envisioned technology as "assistive sidekicks," physical expressive objects that play a supporting role to the AC, ensuring an AC's conversational agency without replacing them or their knowledgeable partner. We then designed a workshop to explore this envisioned technology.

\subsection{Step 2: Co-design Workshop}

We designed an accessible workshop that included three phases: (1) telling to uncover important design goals and parameters, (2) making to build potential sidekick forms, and (3) enacting to design sidekick motions and behaviors. The workshop was 6 hours long.

While co-design workshops provide benefits for aggregating perspectives and expertise in design [49], some design activities can be inaccessible to ACs who have speech and motor disabilities. Co-design activities can heavily rely on the ability of participants to verbally express their ideas in a timely manner and to physically produce sketches or prototypes. We therefore designed a co-design workshop with accessibility in mind, to ensure that ACs' ideas would be included in the process. Our goal was to accommodate the range of speaking rates, styles and preferences of our participants. First, we established everyone as a collaborator and used making as a form of ideation [31]. Next, we developed a series of Access Commitments, following prior work and in collaboration with an SLP [6, 10, 31]. Our Access Commitments included: (1) minimizing participant fatigue by planning three breaks between each phase and allowing asynchronous discussion after the workshop, (2) sending question prompts in advance, (3) establishing preferred modes of communication, (4) allowing enough time for everyone to communicate during the workshop, and (5) making visuals and materials easy to reference and within reach for ACs.

Workshop Participants. Workshop participants (for whom we use pseudonyms) included two augmented communicators (ACs), their close conversation partners, and professional puppeteers. Tammy and Matt are both AAC device and wheelchair users (aged 38 and 23 respectively). Tammy participated in the workshop with her dad, and Matt participated with his mother and his aide. Both have cerebral palsy (CP) and more than 18 years of experience using AAC devices. Tammy uses a joystick to point to words on her device and pauses over her target to select. Matt's device constantly scans through all options on the display (e.g., word, letter, shortcut) until he presses his head switch to select a desired option. Matt needs more time to use his device than Tammy. Matt and Tammy also use some nonverbal behaviors to communicate. Tammy uses her hands to make pointing gestures and signs she and her family have agreed on. Matt uses arm restraints because of his $\mathrm{CP}$, but makes use of facial expressions and eye blinks to support communication.

We additionally invited three artists with prior experience in puppetry, art and puppet design. Puppeteers understand nuances of movement and use motion to elicit specific interactions and responses. Puppeteers have previously been involved as movement experts in the design and research of nonverbal robots [35, 52]. 
Telling: Uncovering Challenges \& Goals. The first phase of the workshop aimed to discover ACs' needs and challenges in conversation that would benefit from nonverbal support. We carried out brainstorming and story sharing activities to uncover opportunities and tensions related to communication, and to hear prior successful and/or challenging communication experiences. These served as a first step toward identifying potential goals for a sidekick.

In the first activity (brainstorming), we asked participants to write their answers to the question: "What does communicating mean to me?" and to choose a metaphor they related to communication. We used a selection of "Metaphor Cards" [33] and asked participants to choose one to share with the group. Metaphors can allow participants to think about abstract concepts in new ways, and to create novel discussions on a topic [33]. Metaphor Cards were numbered to allow ACs to easily reference them without pointing. This activity aimed to uncover some of the current tensions, hopes, and challenges in ACs' communication.

The second activity (story sharing) aimed to explore and discuss AC needs with the group. We asked ACs to share stories about their face-to-face conversations which highlighted challenging, successful or humorous moments. This raised discussions about potential conversational goals a sidekick could assist with (Table 1).

Making: Aesthetics \& Use Cases. Drawing from the stories shared, participants prototyped assistive sidekicks on two AC-led teams. The goal was to explore how a sidekick might address the needs and values identified during the telling phase, and to thinkthrough-making by rapidly prototyping ideas in a group [42]. We asked participants to select one challenge that resonated with them from the discussions, and to use craft materials to create an assistive sidekick that might alleviate the identified challenge. We provided materials to create basic structures, and decorative material such as fabric, feathers, and googly eyes. We divided participants into two teams, each led by an AC and including their caregiver and one or two puppeteer(s). After prototyping, teams demonstrated their sidekicks, described the challenge it would alleviate and the behaviors it could leverage to do so.

Enacting: Developing Motions \& Behaviors. The third part of the workshop aimed to design sidekick motions and behaviors that could meet specific goals. Puppeteers enacted situations and behaviors using improvisation as a form of bodystorming [50] to explore how previously identified AC needs would play out through motion and behavior. Similar improvisation with domain experts has been previously used in HRI research to explore motion for robotic prototypes $[51,52,59]$. We guided puppeteers through a behavior improvisation session that built upon what they had learned from ACs and their partners to generate concrete behaviors for supportive nonverbal sidekicks.

To minimize AC participants' fatigue we decided not to include ACs in this last phase of the workshop. Only puppeteers participated in enacting specific sidekick motions. Researchers sent videos of the generated behaviors to ACs for their feedback post-workshop.

The researchers selected four identified conversational goals for the puppeteers to explore, based on their prominence in discussion and their diversity. The selected goals are bolded in Table 1. One goal at a time, we instructed puppeteers to generate as many behaviors as they could to achieve it. For example, puppeteers were instructed to show many ways in which a sidekick might communicate to the conversation partner that the $\mathrm{AC}$ would like to add something to the conversation. After exploring possible appropriate motion gestures for each goal, we held a brief discussion with puppeteers to talk about what worked, what did not, and what they noticed in the process of improvising motion to support nonverbal supportive goals for sidekicks.

Data Collection and Analysis. All research procedures were approved by our university's institutional review board. All AC participants were recruited via AAC interest group email lists. Puppeteers were recruited through art school referrals. Workshop participants were compensated with 20 USD per hour. Survey participants were compensated with a 30 USD gift card.

We captured video, audio and photographs during the workshop. Three paper authors additionally took observational notes including participant quotes and paraphrasing of events with timestamps. We used Affinity Diagramming to perform thematic analysis [38] on the notes post-workshop. To analyze the behavior improvisations, we focused on identifying emergent themes from the puppeteergenerated motion, also using thematic coding. To evaluate how accessible our workshop was, we examined ACs' participation by evaluating the turns they took as measured in prior work [58]. We also directly asked ACs about their workshop experience and inquired suggestions for improvement.

\section{WORKSHOP FINDINGS}

The workshop resulted in several findings: (1) Design parameters to guide the design of nonverbal communication sidekicks: attention, precision, and timing in communication; (2) a refined set of AC conversational goals that a sidekick might support (Table 1); (3) a set of physical prototypes to represent potential sidekick forms (Figure 3); and (4) a set of motions and behaviors that the sidekick could use (Table 2). These four findings tackle four layers of this design space, one building on top of the other. Together, they form a coherent picture of how one might design robotic sidekicks to support ACs' nonverbal behavior.

\subsection{Attention, Precision, Timing: Parameters for Nonverbal Communication}

We begin by defining the design parameters that manifested throughout our workshop. These parameters do not encapsulate all challenges ACs face, but can serve as design guidelines to be explicitly considered when designing sidekicks for AAC.

The attention parameter is concerned with ACs' need to be able to draw attention to themselves, to divert attention towards something other than themselves, and to maintain their partner's attention during an interaction. Our ACs reported struggling with calling attention to themselves or fully participating in conversation because of motor and speech impairments. They reported that it is difficult to produce a loud sound, raise a hand to participate, or point to something-an attention challenge that echos prior work [56]. Codesigners expressed excitement about the sidekick's potential to use motion to call for attention during communication. Participants shared how they could use the sidekick to signal for attention in crowded and loud places by waiving at others or giving others a funny look. Caregivers and ACs discussed that being able to divert 
attention towards something other than the $\mathrm{AC}$ was also important. One of the caregivers commented: "[when there is a long silence] the person over here is like, can I come over to the other side and look?" Her comment sparked a discussion on a potential use of a sidekick's nonverbal behavior to divert a conversation partners' attention to ACs' screens. However, ACs also expressed hesitation about drawing too much attention, noting that sidekicks should balance bringing attention to the ACs while at the same time not being "too distracting."

The precision parameter describes two conversational functions: (1) the need to add nuance to a spoken message to more precisely match an AC's intention and (2) the need to add clarity to the AC's existing nonverbal communication. Participants suggested that a sidekick could help convey the AC's tone and mood when needed, like acting in a playful way to convey lightheartedness. Co-designers also discussed how ACs' nonverbal communication can be faster than speaking with the AAC device, but also more ambiguous. Tammy explained: "One day my mom taught me to point to my throat when I was thirsty. I was thirsty so I tried my new sign on dad and he got a funny look on his face. He rubbed [ointment] all over my neck, it was a while before I got a drink." Thus, sidekick nonverbal communication should be precise, as an ambiguous message can come at a great cost and be difficult to repair.

The timing parameter describes the level at which communication can be conveyed in the exact moment that is needed. Being able to say things in time is a challenge for ACs, given that operating an AAC device is slower than producing verbal speech. ACs expressed a need to be able to control timing, such as when to take a turn during a conversation, or when to ask for additional time. Matt's mother reported: "Matt has so much stuff pre-programmed in there [AAC device], but the problem is that he can't get to it very quickly so it doesn't work even though it is already there... he can't get there fast enough". To address Matt's communication timing issue, Matt's family created a light that is placed on his wheelchair, close to his head. Matt turns the light on with a head switch when he is ready to speak, for example, in his college classes. Matt's light is one example that allows him more control over the timing of his communication by non-verbally alerting other speakers. In addition, the timing of when a sidekick displays a nonverbal behavior, either before the AC speaks or during their turn as to accompany their message, was further explored during puppeteer improvisations.

In the next section we describe how these three design parameters were manifested in each stage of the sidekick co-design process.

\subsection{Conversational Goals for Sidekicks}

The first two workshop phases (Telling and Making) allowed us to construct and refine a list of possible conversational goals for a robotic sidekick (Table 1). These goals address conversational and social challenges experienced by ACs and partners when having a conversation involving AAC.

Stories shared by close conversation partners elaborated on ACs' communication challenges. For example, Matt's mom explained how even though she is familiar with AAC devices, some words still sound harsh: "I was with an AAC user and we were having a talk. We were doing something, and she said "I have got to go now. Goodbye." I found myself feeling hurt when she said it. There is an aspect of
Table 1: Goals along with their corresponding numbers and the stage at which they were introduced: original goal from formative work $(\mathrm{O})$, goal mentioned during the telling phase (T), goal mentioned in making (M). Goals $1,5,8$, and 10 were selected to be further explored in the enacting phase.

\begin{tabular}{lll}
\hline$\#$ & Stage & Goal \\
\hline $\mathbf{1}$ & $\mathbf{O}$ & Show others the AC wants to participate \\
2 & $\mathrm{O}$ & Encourage addressing the AC directly \\
3 & $\mathrm{O}$ & Show others there is an AAC device problem \\
4 & $\mathrm{O}$ & Show others the AC needs more time \\
$\mathbf{5}$ & $\mathbf{T}$ & Fill the silence gap while the AC types \\
6 & $\mathrm{~T}$ & Show others the AC is ready to communicate \\
7 & $\mathrm{~T}$ & Tell others to remember what they're talking about \\
$\mathbf{8}$ & $\mathbf{M}$ & Soften an AAC device message \\
9 & $\mathrm{~T}$ & Show others that the AC is typing \\
$\mathbf{1 0}$ & $\mathbf{T}$ & Invite others to look at the AC's screen \\
11 & $\mathrm{~T} / \mathrm{M}$ & Show others the AC disagrees \\
12 & $\mathrm{M}$ & Add humor to an AAC device message \\
13 & $\mathrm{M}$ & Convey emotion quickly \\
\hline
\end{tabular}

the computer that made it abrupt." This experience is related to the parameter of precision. The verbal communication from the AAC imprecisely conveyed the AC's feelings, so Matt's mom did not know if the AC meant to say goodbye in a warm or cold way. An accompanying nonverbal signal might have added precision to the verbal utterance by augmenting it expressively.

Tammy's father described another challenge: the difficulty of remembering a conversational topic due to the time it takes ACs to craft a message. He explained that Tammy's subtle head movement is a useful cue to know when to remember the topic of conversation: "I tell others to watch when Tammy looks down, that means she is typing, and we need to remember this is what we are currently talking about." Tammy's natural movement of lowering her head serves as a timely marker. Nonetheless, it requires an attentive partner who is familiar with Tammy's nonverbal behavior to be able to recognize this gesture.

These examples, along with other stories told by ACs and their caregivers highlighted many opportunities for nonverbal behaviors to support ACs' communication and agency (Table 1). We used the identified needs list as a "design space guide" for the next exploration stage of our work. Furthermore, we hope some of the needs identified here will guide future work on the design of nonverbal robotic sidekicks for AAC.

\subsection{Sidekick Aesthetics \& Use Cases}

The prototyping session during the workshop's Making phase turned the focus to an assistive sidekick's appearance and function, while setting aside concerns of technical feasibility. In this activity, form followed function as the teams first decided on a need from the identified needs list before building it from craft materials. The prototype designed by Tammy's team (Figure 3, left) had anthropomorphic features such as eyes, eye brows, and hair, while Matt's (Figure 3, right) resembled more of a "protest sign" object with a message that would move and appear as needed. 


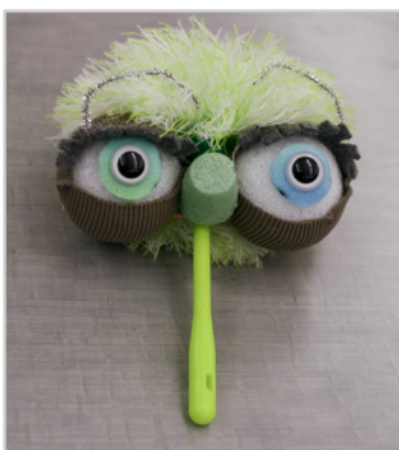

Team Tammy

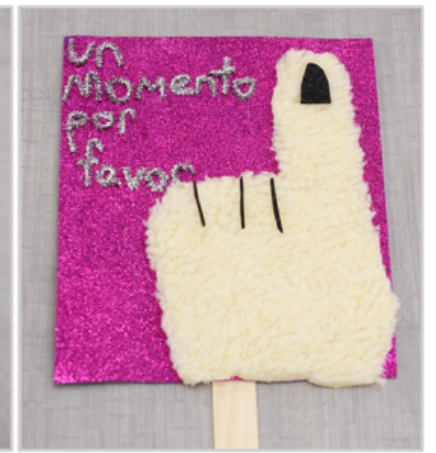

Team Matt

Figure 3: Prototypes by Tammy's (left) and Matt's teams (right). Tammy's prototype featured a large set of eyes, customized to express sentiment. Matt's prototype read "one moment please" to communicate a desire to contribute.

Adding Emotion Through Facial Gestures. Tammy's team decided to address the need to convey emotions quickly in times when it is difficult to use an AAC device, like in noisy and crowded places. This design goal touched primarily on the attention and precision parameters. The sidekick was intended to capture and express Tammy's sense of humor in communication (add precision to her messages) and to be visible from different angles (to grab attention). The team prototyped an expressive face, considering gaze and eyebrows as legible ways to quickly convey emotion. Tammy's dad mentioned how the eyes could be shortcuts for expressions (e.g., rolling eyes or "bored" droopy eyes). Tammy playfully said she wanted to use her sidekick to express "you are pissing me off". Tammy's team spent time customizing the prototype's form to represent Tammy, for example matching its eye color to Tammy's eyes.

Signaling Disagreement Through Object Appearance. Matt was primarily interested in having a way to express that he disagreed and needed a moment to share his point of view. To accomplish this goal, Matt's team designed an object that resembled a protest sign, carefully designed to show respectful disagreement. Matt's goal touched upon all three of the design parameters. In his optimal scenario, the sidekick would convey that Matt disagrees in a precise way that does not seem disrespectful. It would draw attention to let others know he wants to contribute. Finally, it would use the right timing and give Matt time to prepare a response. In order to convey respectful disagreement, the team used a magentaglitter background and furry fabric, which served as a lighthearted visual signal that aims to soften the message. The use of a different language ("Un momento por favor" in Spanish) also increases playfulness. To achieve drawing attention towards Matt, the team used bold colors, and a shape of a pointing finger with its nail painted black. If necessary, the prototype could rest close to Matt's face, pointing directly at him for additional attention and lowering itself to a hidden position when not in use.

\subsection{Sidekick Behaviors and Motions}

In the final workshop activity (Enacting), puppeteers improvised different behaviors to meet four conversational goals identified in prior activities (Table 1), with the newly generated prototypes. The selected goals were: (1) filling the silence gap while an AC types and others wait, (2) showing the AC's intent to participate, (3) softening a message spoken by the AC, and (4) inviting others to look at the AC's screen. We explored these few goals in-depth as an initial examination of this complex design space, prioritizing a deeper exploration of possible behaviors per goal. In future work we would like to examine additional needs and goals. Puppeteers improvised using the prototypes created by Tammy and Matt, but sometimes also used their own bodies or other props. In this section we describe each explored goal, the motions used to achieve it, and the motion patterns that emerged.

The puppeteer improvisations shared a common structure, composed of two "stages" presented in sequence. First, they performed a motion to call for attention. Such motions included making the sidekick take up more space by moving upwards or outwards, spinning or moving quickly, or appearing from a hidden place. After attention was captured, puppeteers tried to convey the main message or purpose of the gesture by either mirroring an action that the $\mathrm{AC}$ would be doing (e.g., mimicking typing on a keyboard) or by demonstrating something that they would like the conversation partner to do (e.g., look at the AAC device screen to invite the partner to do the same).

The motions improvised by puppeteers also reflected our identified design parameters. First, attention was a key function for motion that preceded conveying a message. The sidekick's movement and physical presence supported drawing attention to the AC. The second part of the behavior, either mirroring the AC or suggesting what the conversation partner should do, served to clarify the message and make it more precise. These behaviors also considered the right timing by presenting themselves either in response to an AC's action (e.g., while the AC typed) or in parallel, as the AC spoke their message. Puppeteers generated 10 different behaviors for each goal, a total of 40 different behaviors. We include three example behaviors for each goal in Table 2 .

In order to fill the silence gap that occurs when an AC types and a partner needs to wait for a response, puppeteers made sidekicks move rhythmically in circles (like a "loading" icon) and mimicked typing on a keyboard to show progress. Other explored behaviors aimed to fill the silence through entertainment, such as having the sidekick give the partner a back massage or dance and sing. The precision parameter was prioritized for this goal-puppeteers agreed that more familiar gestures like typing or "loading" were easier to understand.

To show that the AC wants to participate the puppeteers had to first find an effective way of interrupting an ongoing group conversation (played by other puppeteers). Timing was revealed as the most complex for this goal-it was difficult for puppeteers to identify a good time to interrupt a busy group. Some motions that explored ways to call a group's attention included moving the sidekick upwards so that everyone could see it, making the sidekick appear in a sudden manner, or moving outwards to a central focal point of the group. Once the prototype caught the group's attention, 
Table 2: Puppeteers generated 10 behaviors for each selected goal using sidekick prototypes of their choice. We highlight three example behaviors per goal.

\begin{tabular}{|c|c|c|}
\hline Goal & Behavior Title & Behavior Description \\
\hline \multirow[t]{4}{*}{ Fill silence gap } & Tapper & Taps fingers to show typing \\
\hline & Dancer & Dances and sings \\
\hline & Boomerang & Moves cyclically out from the \\
\hline & & $\mathrm{AC}$ and back to original position \\
\hline \multirow[t]{4}{*}{ Let $\mathrm{AC}$ participate } & Up Up Up & Moves up for attention, then \\
\hline & & $\begin{array}{l}\text { opens mouth when partners } \\
\text { look at the AC }\end{array}$ \\
\hline & Attention Sign & Springs up sign from flat to upright \\
\hline & Outward Reach & $\begin{array}{l}\text { Moves forward for attention, gazes } \\
\text { at partners who are still speaking }\end{array}$ \\
\hline \multirow[t]{3}{*}{ Soften a message } & Gentle "No" & Spins to soften "no" \\
\hline & It’s Time & $\begin{array}{l}\text { Looks at watch patiently to } \\
\text { soften "I have to go" }\end{array}$ \\
\hline & Bye-bye Wave & Waves to soften "I have to go" \\
\hline \multirow{3}{*}{$\begin{array}{l}\text { Invite partner to } \\
\text { look at screen }\end{array}$} & Imitate Me & Demonstrates looking at screen \\
\hline & Inviting Arm & $\begin{array}{l}\text { Gestures with an arm to invite } \\
\text { partner to look at screen }\end{array}$ \\
\hline & Swirly Look & Spins then looks at screen \\
\hline
\end{tabular}

the puppeteers attempted to communicate the intent by pointing at the AC, gently poking the partner, or by using gaze to look at the $\mathrm{AC}$ to indicate with precision that the $\mathrm{AC}$ wants to participate.

The third goal explored how a sidekick could soften a message. While nonverbal behavior allows people to set the tone of a verbal message, monotonic AAC device speech can make this challenging. Puppeteers explored adding emotion to a message through slow motion, head tilts, and nodding. To soften the message even more, puppeteers used signals of empathy, like sending a kiss or patting the partner's back. Expressing emotion allowed clearer communication of intention. Being able to soften a verbal message is primarily concerned with precision - the motion is intended to communicate the AC's intention more accurately. Timing was connected to the level of precision-varying when and at what rate motions are presented could alter the perceived level of "softness" or "harshness."

The last explored goal intended to invite a communication partner to look at the AAC device's screen. Similarly to other goals, puppeteers achieved this in two steps: catching the partner's attention and then making a gesture towards the screen. Different motions successfully captured the partner's attention, like spinning the sidekick or making it appear from behind the screen. Once the sidekick had the partner's attention, it could indicate for them to come and look at the screen. This was achieved by pointing the sidekick towards the screen, using gaze, or using the sidekick's head to point. Achieving precision was challenging for this goal, as it did not necessarily accompany a verbal message by the AC, but rather communicated its own message.

We shared videos of the puppeteers' improvised behaviors with our two AC participants after the workshop. Only Matt provided additional input. Behaviors that were "straightforward," as described by Matt, were positively rated such as the Tapper, Attention Sign, Inviting Arm, and It's Time. Other behaviors were labeled "too distracting," like the Dancer, and were therefore disliked.

\section{WORKSHOP ACCESSIBILITY REFLECTIONS}

As an additional contribution, we report on the successes and challenges of our five access commitments (Section 3.2).

Our first commitment aimed to minimize participant fatigue by planning breaks between activities and allowing asynchronous discussion post-workshop. This had both positive effects and challenges. Breaks were used and appreciated by all. Despite our attempts, there was less engagement post-workshop, with only Matt responding with feedback about puppeteer-generated behaviors. Matt uses his computer every day for work which was not the case for Tammy. We recommend future work to acknowledge differences in computer usage, and to tailor post-activity follow-up strategies for each participant's usage context.

The second commitment was to send question prompts that will be used in the workshop in advance. Both AC participants shared stories that were pre-recorded on their device, suggesting they made use of the prompts that were sent in advance.

Our third commitment allowed time to establish preferred modes of communication. We found that asking participants to share communication preferences and strategies at the beginning of the workshop prevented possible misunderstandings. Matt's mom explained that Matt uses blinks to communicate "yes", "no" and "I don't know." Matt's team then used these cues when working on the prototype. Matt's mom also explained that Matt gives 1-2 word answers and asks others to elaborate on his behalf. Matt used this strategy in the workshop to ask his mother to elaborate for him. Similarly, knowing that Tammy puts her head down to indicate that she wants to type, as her father explained, helped a puppeteer unfamiliar with AAC realize that Tammy was busy typing and that she should wait before asking her next question.

Our fourth commitment was to allow everyone enough time to communicate during the workshop. We took turns in an established order, which supported ACs and gave them more time to communicate. For each activity, all participants took at least one turn. One strategy that emerged was to first ask Matt and Tammy the question, but then let other participants take their turns as Matt and Tammy prepared an answer. This encouraged longer responses from AC participants. Another emergent strategy was for each team to confirm all design decisions with the ACs. Matt's team asked Matt a series of questions for feedback. For instance, after Matt said "I want to use what we were talking about," the team asked follow up questions and, with Matt's blinking to answer yes or no, narrowed the topic down to find out what Matt meant. Tammy created a sketch while her team consulted with her on key decisions.

Our fifth commitment was to make all materials easy to reference and access. Labelling visual materials with numbers and annotating discussions on a board enabled ACs to use verbal shortcuts to refer to specific concepts. For example, Matt used a number to indicate his chosen metaphor instead of having to type out the metaphor name. To support physical access, we used adjustableheight tables that allowed ACs to get close to prototyping materials. However, we lacked other useful adaptive tools (e.g., head-mounted tools) which may have eased prototyping. We recommend labelling all visual materials and asking participants about desired access tools in the process of designing a workshop. 


\section{DISCUSSION \& FUTURE WORK}

We have identified opportunities in which socially assistive robots could augment AAC-based communication using motion. Robotic sidekicks are not the only solution, but provide an interface to explore motion-based nonverbal communication, which is missing from current speech-based AAC technology. In this section, we reflect on how social robots' motions could improve precision, timing, and attention in communication, and identify further research questions that can guide the design of functional robotic sidekicks.

\subsection{Precision through Embodied Motion}

The sidekick's motions should be immediately recognizable and interpretable so that people who may not be familiar with AAC or an AC's communication style can still understand the message [8] Most behaviors produced by puppeteers in our workshop leveraged common, established nonverbal gestures to convey a particular concept. These included gaze and eye motion, head orientation, pointing, and iconic gestures such as looking at a watch to communicate "time." Leveraging such "universal symbols" was helpful for quick communication that can speak to a range of audiences, as puppeteers explained when they reflected on common gestures. That said, gestures are likely to be culture-specific, and would need to be re-examined depending on the user and audience. Precision through embodied motion is only one approach; other modalities, such as expressive voices [15], may add further precision.

We learned that specific aesthetics can also support conveying a precise message. Tammy's team created an anthropomorphic sidekick with highly visible facial features that could express familiar facial expressions. Matt's sidekick, shaped as a sign, suggested a strong and direct presence, though it also used its aesthetics to convey friendliness. Future work could examine visual aesthetics of sidekicks and AAC technology as an additional modality for increasing the precision of a message. The final designs may have been shaped by the materials available at the workshop as well as personal preferences, so additional explorations with other materials and AC participants could reveal additional sidekick forms.

\subsection{Timing of Sidekick Behaviors}

The timing of our proposed sidekick behaviors showed both consistency and flexibility. Behaviors followed a consistent two-stage sequence, starting with a motion to call for attention, and then taking some communicative action. However, behavior timing was also flexible depending on the context of the interaction. Some behaviors occurred before the AC verbally communicated, in order to fill a silence gap or to ask the partner to view the AC's screen. Other behaviors overlapped with AC speech, as when the nonverbal behavior was intended to soften a message or increase a message's precision. Finally, some behaviors occurred at short intervals in communication, for example to let the group know that an $\mathrm{AC}$ is ready to speak. Identifying the right time to perform a behavior requires awareness of context and conversational dynamics.

In our workshop, we sidestepped this timing issue by implicitly assuming the sidekick would be activated at the right time. However, leaving the AC to activate a sidekick's behaviors at the right time is likely to increase their cognitive and physical burden. On the other hand, having a sidekick autonomously recognize interaction context to activate its own behaviors is itself a major research challenge. Future work could examine tradeoffs between user-activated and autonomously activated sidekick behaviors.

\subsection{Modalities for Gaining Attention}

Throughout the workshop, it became clear that one of the most important abilities for a sidekick is to gain attention from conversation partners. This was evident not only in the behaviors explored, but also in the physical prototypes created by the co-design teams. Both teams used embodied properties to gain attention in their prototypes: Tammy's team used eyes, a feature that is known to draw attention, and Matt's team used components with high visibilityglittery pink paper and furry fabric. Puppeteers also varied motion speed and gesture size to call for more or less attention as needed, an approach similar to prior work [1].

Matt's current use of his wheelchair light to signify his desire to speak highlights an opportunity for sidekicks to gain attention through more ambient, non-anthropomorphic modalities. This might be especially useful in situations where anthropomorphic motions are inappropriate or impractical. Future work may investigate the benefits of different attention-getting modalities for expressive sidekicks in challenging contexts, like noisy environments.

\subsection{Building Motion-Expressive Sidekicks}

Translating the designs from this workshop into fully functional robots will require additional work and design. For example, the sidekick should have accessible controls (e.g., eye gaze, infrared remote control, or switches) so an AC can trigger different behaviors. It is important that these interfaces do not impose additional burden on ACs. Future work could explore what type of control is most desirable to ACs, potentially extending control to include the partners (as with groupware [16]) or robot autonomy (as with shared autonomy[26]). If the sidekick has any amount of autonomy, it will need to use techniques from sensing and automation to select context-aware behaviors. This is still an open research challenge in social HRI. The sidekick might also use machine learning to personalize its behavior to a single individual over time.

\section{CONCLUSION}

Our findings offer design opportunities to enhance augmented communicators' (ACs') face-to-face conversations by using socially assistive robots as a form of motion-based AAC. In a co-design workshop with ACs, their close conversation partners and puppeteers, we explored the metaphor of a "sidekick"-a physical device that supports ACs in conversation through movement. We provide evidence of the usefulness of motion to bring attention to ACs, convey precise messages, and enable ACs to participate in conversation at the right time. We present key needs that sidekicks could successfully address, as well as accessible co-design commitments that amplify ACs' participation in co-design.

\section{ACKNOWLEDGMENTS}

We thank our anonymous reviewers and participants. Research is supported by NSF grant IIS-1755823 and NSF grant SES-1734456. 


\section{REFERENCES}

[1] Lucy Anderson-Bashan, Benny Megidish, Hadas Erel, Iddo Wald, Guy Hoffman, Oren Zuckerman, and Andrey Grishko. 2018. The greeting machine: an abstract robotic object for opening encounters. In 2018 27th IEEE International Symposium on Robot and Human Interactive Communication (RO-MAN). IEEE, 595-602.

[2] Sean Andrist, Xiang Zhi Tan, Michael Gleicher, and Bilge Mutlu. 2014. Conversational Gaze Aversion for Humanlike Robots. In Proceedings of the 10th International Conference on Human-Robot Interaction (HRI). ACM.

[3] Shiri Azenkot, Catherine Feng, and Maya Cakmak. 2016. Enabling building service robots to guide blind people a participatory design approach. In 2016 11th ACM/IEEE International Conference on Human-Robot Interaction (HRI). IEEE, 3-10.

[4] Dare A Baldwin. 1995. Understanding the link between joint attention and language. Foint attention: Its origins and role in development (1995), 131-158.

[5] Erin Beneteau. 2020. Who Are You Asking ?: Qualitative Methods for Involving AAC Users as Primary Research Participants. (2020), 1-13.

[6] Cynthia L Bennett, Kristen Shinohara, Brianna Blaser, Andrew Davidson, and Kat M Steele. 2016. Using a Design Workshop To Explore Accessible Ideation. In Proceedings of the 18th International ACM SIGACCESS Conference on Computers and Accessibility. ACM, 303-304.

[7] David R Beukelman, Pat Mirenda, et al. 1998. Augmentative and alternative communication. Paul H. Brookes Baltimore.

[8] Sarah W Blackstone. 1999. Circles of communication partners. Augmentative Communication News 12 (1999), 1-2.

[9] Eva Brandt, Thomas Binder, and Elizabeth BN Sanders. 2012. Ways to engage telling, making and enacting. Routledge international handbook of participatory design. Routledge, New York (2012), 145-181.

[10] Robin N. Brewer. 2018. Facilitating Discussion and Shared Meaning: Rethinking Co-Design Sessions with People with Vision Impairments. In Proceedings of the 12th EAI International Conference on Pervasive Computing Technologies for Healthcare (New York, NY, USA) (PervasiveHealth '18). Association for Computing Machinery, New York, NY, USA, 258-262. https://doi.org/10.1145/3240925.3240981

[11] Jessica R Cauchard, Kevin Y Zhai, Marco Spadafora, and James A Landay. 2016 Emotion encoding in human-drone interaction. In 2016 11th ACM/IEEE International Conference on Human-Robot Interaction (HRI). IEEE, 263-270.

[12] Sarah Collins, Ivana Markova, and Joan Murphy. 1997. Bringing conversations to a close: The management of closings in interactions between AAC users and 'natural'speakers. Clinical linguistics \& phonetics 11, 6 (1997), 467-493.

[13] Lieven De Couvreur and Richard Goossens. 2011. Design for (every) one: cocreation as a bridge between universal design and rehabilitation engineering. CoDesign 7, 2 (2011), 107-121.

[14] Paul Dourish. 2004. Where the action is: the foundations of embodied interaction. MIT press.

[15] Alexander Fiannaca, Jon Campbell, Ann Paradiso, and Meredith Ringel Morris 2018. Voicesetting: Voice Authoring UIs for Improved Expressivity in Augmentative Communication. (2018). https://doi.org/10.1145/3173574.3173857

[16] Alexander Fiannaca, Ann Paradiso, Mira Shah, and Meredith Ringel Morris. 2017 AACrobat: Using mobile devices to lower communication barriers and provide autonomy with gaze-based AAC. In Proceedings of the 2017 ACM Conference on Computer Supported Cooperative Work and Social Computing. ACM, 683-695.

[17] Christopher Frauenberger, Katta Spiel, and Julia Makhaeva. 2019. Thinking OutsideTheBox - Designing Smart Things with Autistic Children. International fournal of Human-Computer Interaction 35, 8 (2019), 666-678. https://doi.org/10. 1080/10447318.2018.1550177

[18] Julia Galliers, Stephanie Wilson, Abi Roper, Naomi Cocks, Jane Marshall, Sam Muscroft, and Tim Pring. 2012. Words are not enough: Empowering people with aphasia in the design process. ACM International Conference Proceeding Series 1 (2012), 51-60. https://doi.org/10.1145/2347635.2347643

[19] Charles Goodwin. 2011. Contextures of action. Embodied interaction: Language and body in the material world (2011), 182-193.

[20] Joy E Hanna and Susan E Brennan. 2007. Speakers' eye gaze disambiguates referring expressions early during face-to-face conversation. Fournal of Memory and Language 57, 4 (2007), 596-615.

[21] DJ Higginbotham, K Fulcher, and J Seale. 2016. Time and timing in interaction involving individuals with ALS, their unimpaired partners and their speech generating devices. The silent partner (2016), 199-229.

[22] D Jeffery Higginbotham. 2009. In-person interaction in AAC: New perspectives on utterances, multimodality, timing, and device design. Perspectives on Augmentative and Alternative Communication 18, 4 (2009), 154-160.

[23] D Jeffery Higginbotham and DP Wilkins. 1999. Slipping through the timestream: Social issues of time and timing in augmented interactions. Constructing (in) competence: Disabling evaluations in clinical and social interaction 2 (1999), 49-82.

[24] Guy Hoffman, Oren Zuckerman, Gilad Hirschberger, Michal Luria, and Tal ShaniSherman. 2015. Design and evaluation of a peripheral robotic conversation companion. In 2015 10th ACM/IEEE International Conference on Human-Robot Interaction (HRI). IEEE, 3-10.
[25] Hiroshi Ishii. 2008. Tangible bits: beyond pixels. In Proceedings of the 2nd international conference on Tangible and embedded interaction. $\mathrm{xv}-\mathrm{xxv}$.

[26] Shervin Javdani, Henny Admoni, Stefania Pellegrinelli, Siddhartha S. Srinivasa, and J. Andrew Bagnell. 2018. Shared Autonomy via Hindsight Optimization for Teleoperation and Collaboration. International fournal of Robotics Research 37 (2018), 717-742. Issue 7

[27] Shaun K. Kane, Meredith Ringel Morris, Ann Paradiso, and Jon Campbell. 2017. At Times Avuncular and Cantankerous, with the Reflexes of a Mongoose: Understanding Self-Expression Through Augmentative and Alternative Communication Devices. In Proceedings of the 2017 ACM Conference on Computer Supported Cooperative Work and Social Computing (Portland, Oregon, USA) (CSCW'17). ACM, New York, NY, USA, 1166-1179. https://doi.org/10.1145/2998181.2998284

[28] Mark L Knapp, Judith A Hall, and Terrence G Horgan. 2013. Nonverbal communication in human interaction. Cengage Learning.

[29] Heather Knight, Timothy Lee, Brittany Hallawell, and Wendy Ju. 2017. I get it already! the influence of chairbot motion gestures on bystander response. In 2017 26th IEEE International Symposium on Robot and Human Interactive Communication (RO-MAN). IEEE, 443-448.

[30] Spyros Kousidis and David Schlangen. 2015. The power of a glance: Evaluating embodiment and turn-tracking strategies of an active robotic overhearer. In 2015 AAAI Spring Symposium Series.

[31] Amanda Lazar, Jessica L Feuston, Caroline Edasis, and Anne Marie Piper. 2018. Making as expression: Informing design with people with complex communication needs through art therapy. In Proceedings of the 2018 CHI Conference on Human Factors in Computing Systems. ACM, 351.

[32] Hee Rin Lee, Selma Šabanović, Wan-Ling Chang, Shinichi Nagata, Jennifer Piatt, Casey Bennett, and David Hakken. 2017. Steps Toward Participatory Design of Social Robots: Mutual Learning with Older Adults with Depression. In Proceedings of the 2017 ACM/IEEE International Conference on Human-Robot Interaction (Vienna, Austria) (HRI '17). Association for Computing Machinery, New York, NY, USA, 244-253. https://doi.org/10.1145/2909824.3020237

[33] Dan Lockton, Devika Singh, Saloni Sabnis, Michelle Chou, Sarah Foley, and Alejandro Pantoja. 2019. New Metaphors: A Workshop Method for Generating Ideas and Reframing Problems in Design and Beyond. In Proceedings of the 2019 on Creativity and Cognition. ACM, 319-332.

[34] Michal Luria. 2018. Designing robot personality based on fictional sidekick characters. In Companion of the 2018 ACM/IEEE International Conference on Human-Robot Interaction. ACM, 307-308.

[35] Michal Luria, Guy Hoffman, Benny Megidish, Oren Zuckerman, and Sung Park. 2016. Designing Vyo, a robotic Smart Home assistant: Bridging the gap between device and social agent. In 2016 25th IEEE International Symposium on Robot and Human Interactive Communication (RO-MAN). IEEE, 1019-1025.

[36] Yoichi Matsuyama, Iwao Akiba, Shinya Fujie, and Tetsunori Kobayashi. 2015. Four-participant group conversation: A facilitation robot controlling engagement density as the fourth participant. Computer Speech \& Language 33, 1 (2015), 1-24.

[37] David McNeill. 1992. Hand and Mind: What Gestures Reveal about Thought. The University of Chicago Press, Chicago.

[38] Matthew B Miles, A Michael Huberman, Michael A Huberman, and Michael Huberman. 1994. Qualitative data analysis: An expanded sourcebook. sage.

[39] Karyn Moffatt, Joanna McGrenere, Barbara Purves, and Maria Klawe. 2004. The participatory design of a sound and image enhanced daily planner for people with aphasia. In Proceedings of the SIGCHI conference on Human factors in computing systems. 407-414.

[40] Sanika Moharana, Alejandro E Panduro, Hee Rin Lee, and Laurel D Riek. 2019. Robots for joy, robots for sorrow: community based robot design for dementia caregivers. In 2019 14th ACM/IEEE International Conference on Human-Robot Interaction (HRI). IEEE, 458-467.

[41] Bilge Mutlu, Toshiyuki Shiwa, Takayuki Kanda, Hiroshi Ishiguro, and Norihiro Hagita. 2009. Footing in human-robot conversations: how robots might shape participant roles using gaze cues. In Proceedings of the 4th ACM/IEEE international conference on Human robot interaction. ACM, 61-68.

[42] Don Norman. 2013. The design of everyday things: Revised and expanded edition. Basic books.

[43] Joohee Park, Young Woo Park, and Tek Jin Nam. 2014. Wrigglo: Shape-changing peripheral for interpersonal mobile communication. Conference on Human Factors in Computing Systems - Proceedings (2014), 599-602. https://doi.org/10.1145/ 2559206.2574783

[44] Graham Pullin and Shannon Hennig. 2015. 17 ways to say yes: Toward nuanced tone of voice in AAC and speech technology. Augmentative and Alternative Communication 31, 2 (2015), 170-180.

[45] Albert B Robillard. 1994. Communication problems in the intensive care unit. Qualitative Sociology 17, 4 (1994), 383-395.

[46] Albert B Robillard. 1996. Anger in-the-social-order. Body \& Society 2, 1 (1996), $17-30$

[47] Ben Robins, Kerstin Dautenhahn, and Paul Dickerson. 2009. From isolation to communication: a case study evaluation of robot assisted play for children with autism with a minimally expressive humanoid robot. In 2009 Second International 
Conferences on Advances in Computer-Human Interactions. IEEE, 205-211.

[48] Elizabeth B-N Sanders, Eva Brandt, and Thomas Binder. 2010. A framework for organizing the tools and techniques of participatory design. In Proceedings of the 11th biennial participatory design conference. 195-198.

[49] Elizabeth B-N Sanders and Pieter Jan Stappers. 2008. Co-creation and the new landscapes of design. Co-design 4, 1 (2008), 5-18.

[50] Dennis Schleicher, Peter Jones, and Oksana Kachur. 2010. Bodystorming as embodied designing. interactions 17, 6 (2010), 47-51.

[51] David Sirkin and Wendy Ju. 2014. Using embodied design improvisation as a design research tool. In Proceedings of the international conference on Human Behavior in Design (HBiD 2014), Ascona, Switzerland.

[52] David Sirkin, Brian Mok, Stephen Yang, and Wendy Ju. 2015. Mechanical ottoman how robotic furniture offers and withdraws support. In Proceedings of the Tenth Annual ACM/IEEE International Conference on Human-Robot Interaction. 11-18.

[53] David Sirkin, Gina Venolia, John Tang, George Robertson, Taemie Kim, Kor Inkpen, Mara Sedlins, Bongshin Lee, and Mike Sinclair. 2011. Motion and attention in a kinetic videoconferencing proxy. Lecture Notes in Computer Science (including subseries Lecture Notes in Artificial Intelligence and Lecture Notes in Bioinformatics) 6946 LNCS, PART 1 (2011), 162-180. https://doi.org/10.1007/978-3-642-23774$4 \_16$

[54] Kiley Sobel, Alexander Fiannaca, Jon Campbell, Harish Kulkarni, Ann Paradiso, Ed Cutrell, and Meredith Ringel Morris. 2017. Exploring the Design Space of AAC Awareness Displays. In Proceedings of the 2017 CHI Conference on Human
Factors in Computing Systems. ACM, 2890-2903.

[55] Katta Spiel, Christopher Frauenberger, O. S. Keyes, and Geraldine Fitzpatrick. 2019. Agency of autistic children in technology research - A critical literature review. ACM Transactions on Computer-Human Interaction 26, 6 (2019). https: //doi.org/10.1145/3344919

[56] Gabriele B Sweidel. 1991. Management strategies in the communication of speaking persons and persons with a speech disability. Research on Language \& Social Interaction 25, 1-4 (1991), 195-214.

[57] Hamish Tennent, Solace Shen, and Malte Jung. 2019. Micbot: A periphera robotic object to shape conversational dynamics and team performance. In 2019 14th ACM/IEEE International Conference on Human-Robot Interaction (HRI). IEEE, 133-142.

[58] Stephanie Valencia, Amy Pavel, Jared Santa Maria, Seunga (Gloria) Yu, Jeff Bigham, and Henny Admoni. 2020. Conversational Agency in Augmentative and Alternative Communication. In Proceedings of the 2020 CHI Conference on Human Factors in Computing Systems. ACM.

[59] Oren Zuckerman, Dina Walker, Andrey Grishko, Tal Moran, Chen Levy, Barak Lisak, Iddo Yehoshua Wald, and Hadas Erel. 2020. Companionship Is Not a Function: The Effect of a Novel Robotic Object on Healthy Older Adults' Feelings of "Being-Seen". Proceedings of the ACM CHI Conference on Human Factors in Computing Systems (CHI '20) (2020), 1-14. https://doi.org/10.1145/3313831. 3376411 